\title{
Mechanisms and scalings of energetic ion transport via tokamak microturbulence
}

\author{
T. Hauff and F. Jenko \\ Max-Planck-Institut für Plasmaphysik, EURATOM Association, 85748 Garching, Germany
}

\begin{abstract}
The turbulent $E \times B$ advection of energetic ions in 3D tokamak geometry is investigated both analytically and numerically. It is shown that orbit averaging (leading to a significant reduction of the diffusivity) is only valid for low magnetic shear. At moderate or high magnetic shear, a rather slow decrease of the diffusivity is found, proportional to $\left(E / T_{e}\right)^{-1}$ or $\left(E / T_{e}\right)^{-1.5}$ for particles with a large or small parallel velocity component, respectively. The decorrelation mechanisms responsible for this behavior are studied and explained in detail. Moreover, it is found that resonances between the toroidal drift of the particles and the diamagnetic drift of the turbulence can lead to an enhancement of the fast ion transport.
\end{abstract}

\section{INTRODUCTION}

The question whether and to what extent energetic ions in a tokamak are advected by background microturbulence has attracted growing interest over the last several years. While there exists a large number of publications about particles with large gyroradii in 2D turbulence, [1-7] the study of fast particles in a turbulent background in 3D toroidal geometry has (re-)gained attention only quite recently.[8-10] These investigations were, in part, motivated by recent experimental investigations at ASDEX Upgrade which showed a fast radial broadening of the current profile driven by off-axis neutral beam injection in the absence of any measurable magnetohydrodynamic activity,[11] and seem to contradict earlier experimental results claiming that such an effect should not exist (see, e.g., Ref. [12]). The present paper wants to shed light on this crucial question (especially for future D-T-based experiments like ITER [13]) by studying different decorrelation mechanisms for passive tracers in a 3D turbulent environment. We shall find that the transport levels generally depend on the turbulence characteristics as well as on the orbit parameters of the fast particles in a very sensitive way. This will enable us to explain both situations showing a fast drop of diffusivity with growing particle energy as well as situations in which the transport remains high for a while until it falls off rather slowly, inversely proportional to the particle energy.

In two dimensions, the question of fast particle transport seems to be solved. Here, a fast particle is simply characterized by its gyroradius $\rho_{g}$ and follows the $E \times B$ drift in the gyroavaraged electrostatic potential.[14] Unfortunately, several earlier publications were inconsistent. While some claimed that the particle diffusivity drops steadily as the gyroradius is increased, [1, 2] others found that the transport level keeps constant for gyroradii up the the correlation length of the fluctuations, [3] or that it even may increase strongly.[4, 5] However, by means of careful systematic studies, it could be demonstrated that, in general, one of the former two cases applies, depending on the Kubo number[15] of the electrostatic potential.[6, 7] For Kubo numbers smaller than unity, the diffusivity is reduced monotonically with growing gyroradius, since gyroaveraging smoothes the potential and therefore reduces the effective drift velocity. In contrast, gyroaveraging increases the correlation lenghts of the potential, which for Kubo numbers larger than unity balances the reduction of the drift velocity and therefore keeps the transport constant for gyroradii up to the correlation length. The existence of a regime exhibiting a distinct increase of the transport could not be confirmed. In addition to that, it was shown in Ref. [7] how a constant drift of the background turbulence affects the transport. This finding will carry over to the 3D case.

In three dimensions, the motion of fast particles is characterized by more than just the gyroradius, of course. Depending on the pitch angle, the particles are passing or trapped (see, e.g., Ref. [16]), and in both cases, their motion can be described - in a field-aligned coordinate system - as a superposition of a "circular" (or slightly elliptical) periodic motion and a constant drift in the binormal direction, in analogy with the usual gyromotion. So we will have to ask whether the effects of gyroaveraging (well understood for the 2D case) will translate into some kind of "orbit averaging" in three dimensions, and to what extent the particle drifts generated by curvature and grad-B drifts (in the 3D case) can lead to effects which resemble those observed in two dimensions. Indeed, it has been claimed in Ref. [17] that gyroaveraging as well as drift orbit averaging both lead to a universal reduction of transport. However, in Ref. [18] it was shown that orbit averaging is not valid if the particle is decorrelated by fast parallel dynamics before it can finish its periodic orbit.

In early 3D simulations, studying the interaction of energetic alpha particles with high toroidal mode number instabilities, it was reported that such modes can indeed cause significant alpha-particle transport. However, this effect was found to strongly depend on the turbulence properties as well as on the device.[19, 20] A further explanation of this behavior was not given. In Ref. [8], alpha particles were modelled as a hot Maxwellian species, and their particle fluxes were determined quasilinearly and nonlinearly. A large increase of the flux of the alphas was reported compared to the particle flux of the thermal background plasma. However, as pointed out in Ref. [10], this interpretation was 
mainly due to the special normalization used in this work. Furthermore, thermal particles were not distinguished from non-thermal ones. In another recent work, [9] beam ions were modelled by means of an asymmetric and anisotropic Maxwellian distribution function with a long tail in the beam direction. In qualitative agreement with earlier 2D results, [7] it was found that the fast particle transport becomes large when the poloidal drift velocity of the particles matches the diamagnetic drift velocity of the background turbulence. Since resonances can exist for energies up to about 10 times the thermal energy, it was shown that the redistribution of the beam ions may remain significant up to that energy. This has also been found in a recent quasilinear study [10] employing a slowing-down distribution function. Interestingly, according to Ref. [9], fast particles interacting with turbulent fields exhibit a rather slow decrease of diffusivity (approximately like $1 / E$ ) instead of the much faster decay observed in quasilinear studies.

It is the aim of this paper to provide a systematic study of the physical mechanisms responsible for the diffusion of fast particles. As will be shown, the transport is governed, in general, by the combination of a number of different effects, including gyroaveraging, orbit averging, resonances with the background drift, and decorrelations caused by parallel or perpendicular orbit motion. Furthermore, we will find that it makes a big difference whether orbit averaging is valid or not. Since we are mainly interested in the basic principles underlying the particle-turbulence interactions in a tokamak, we will represent the turbulent background fluctuations by stochastic fields with realistic physical properties, a method used successfully in previous $2 \mathrm{D}$ studies. [6, 7] The energetic particles, on the other hand, are treated as passive tracers. This approximation seems to be well justified by several previous investigations [8-10] in which no significant differences were found between passive and active treatment of the fast particles in the low-density limit. More specifically, Ref. [10] as well as Ref. [21] find that the passive tracer picture is valid for concentrations up to about $2 \%$. The transport will be characterized in terms of diffusion coefficients $D$ corresponding to individual particle trajectories. While this quantity is generally not the same as $D_{\Gamma}=-\Gamma / \nabla n_{0}$ with $\Gamma=\left\langle\tilde{n} \tilde{v}_{E}\right\rangle$, they are closely linked. In particular, it can be shown that $D$ and $D_{\Gamma}$ coincide for $L_{n}=\left(\nabla n_{0} / n_{0}\right)^{-1}=$ const. [22]

The remainder of this paper is organized as follows. In Secs. II and III, we provide some information about the construction of the equilibrium magnetic field and the fluctuating electrostatic potentials used in this work. In Sec. IV, the validity of orbit averaging is examined, and a number of possible decorrelation mechanisms are discussed. In Sec. V, the latter are investigated for the simple case of a shearless magnetic field. Secs. VI and VII then deal with simulations of energetic ions in a realistic sheared magnetic field. We close with some conclusions in Sec. VIII.

For clarification, we would like to mention that in the present paper, the term "orbit motion" refers to the curvature and grad- $B$ drift motion and does not include the $E \times B$ drift (or the diamagnetic drift). Moreover, unless noted otherwise, SI units are used.

\section{EQUILIBRIUM MAGNETIC AND FLUCTUATING ELECTRIC FIELDS}

In the following, we will consider a simple tokamak with circular, concentric flux surfaces, but with a finite aspect ratio. The respective torus coordinates - minor radius, poloidal and toroidal angle - are denoted, respectively, by $r, \theta$, and $\varphi$. The distance between the magnetic axis and the symmetry axis is denoted by $R_{0}$. The existence of a coordinate system with straight field lines and its mathematical properties have been investigated first in Refs. [23, 24]. Therein, it was shown that a general magnetic field can be written in terms of the so-called Clebsch representation [23] $\mathbf{B}=\nabla \beta \times \nabla \Psi$, where $\Psi$ is often taken to be the poloidal flux and $\beta=q(r) \chi-\varphi$. Here, $\chi$ is a new "poloidal" coordinate chosen such that the field lines are straight in the $\chi-\varphi$ plane. The "safety factor" $q$ is defined, as usual, by the relation

$$
q(r)=\frac{1}{2 \pi} \int_{0}^{2 \pi} \frac{\mathbf{B} \cdot \nabla \varphi}{\mathbf{B} \cdot \nabla \theta} d \theta=\frac{1}{2 \pi} \int_{0}^{2 \pi} \frac{r B_{\varphi}}{R B_{\theta}} d \theta
$$

with $R=R_{0}(1+\epsilon \cos \theta)$ and $\epsilon=r / R_{0}$. The corresponding magnetic field can be written as

$$
\mathbf{B}=B_{\varphi} \mathbf{e}_{\varphi}+B_{\theta} \mathbf{e}_{\theta}=\left(B_{0} R_{0} / R\right)\left(\mathbf{e}_{\varphi}+b_{\theta} \mathbf{e}_{\theta}\right)
$$

where $b_{\theta}$ is assumed to be independent of $R$. Inserting the components of $\mathbf{B}$ into Eq. (1) and integrating over $\theta$, we find the expression

$$
B_{\theta}=\frac{B_{0} R_{0}}{R} \frac{1}{q(r)} \frac{\epsilon}{\left(1-\epsilon^{2}\right)^{1 / 2}} .
$$

Thus, an arbitrary safety factor profile $q(r)$ may be chosen do define the magnetic field. Next, we have to calculate the function $\chi(\theta)$. Since, per definition, the magnetic field lines are straight in the $\chi-\varphi$ plane, we have $q \equiv$ 
$(\mathbf{B} \cdot \nabla \varphi) /(\mathbf{B} \cdot \nabla \chi)$. With the help of $\mathbf{B} \cdot \nabla \chi=\mathbf{B} \cdot \nabla \theta d \chi / d \theta$, we finally obtain the relation $[25]$

$$
\chi=2 \arctan \left(\sqrt{\frac{1-\epsilon}{1+\epsilon}} \tan \frac{\theta}{2}\right) .
$$

In the large aspect ratio limit $(\epsilon \rightarrow 0)$, we have $\chi \rightarrow \theta$, as expected.

Since the turbulent fluctuations in a tokamak plasma are strongly elongated along the magnetic field lines, it is convenient for the construction of fluctuating test potentials to use coordinates which are field-aligned. In the local approximation, [26] one thus obtains

$$
x=r, \quad y=\frac{r_{0}}{q_{0}} \beta, \quad z=\chi .
$$

Assuming periodic perpendicular boundary conditions, we can construct a random electrostatic potential according to

$$
\phi(\mathbf{x}, t)=g(z) \sum_{i=1}^{N} A_{i} \sin \left(k_{x, i} x+k_{y, i} y+\omega_{i} t+\varphi_{i}\right),
$$

which is then mapped back onto a Cartesian spatial grid that is used for calculating the particle trajectories. In order to avoid problems with the parallel boundary conditions [26] for our test potentials, for simplicity, we either consider a shearless case, $q(r) \equiv 1.4$, in which the magnetic field lines are closed after 5 poloidal (or 7 toroidal) turns - or we choose the envelope function in Eq. (6) to be $g(z)=0.5 \cos (z-\sin (z))+0.5$ which is quite realistic and renders the parallel boundary conditions at $z= \pm \pi$ irrelevant. In the $y$ direction, the box length is chosen to be $2 \pi r_{0} / q_{0}$, reflecting the real periodicity of the torus. However, provided one is careful, it is also possible to cover the torus by $M$ copies of a flux tube whose width in the $y$ direction is only $2 \pi r_{0} /\left(M q_{0}\right)$. A discussion of this procedure is presented below, in Sec. VI.B.

The physical parameters characterizing the magnetic field and the test potentials in the present study are inspired by the ITER project.[13] Thus, we use $R_{0}=6.2 \mathrm{~m}$ and $B_{0}=5.3 \mathrm{~T}$. For the $q$ profile, we either use $q(r) \equiv 1.4$ or $q(r)=0.5 r^{2}+1.25$. The particles start at a radial position near $r_{0}=0.7 \mathrm{~m}$. The stochastic electrostatic potential is created according to Eq. (6) such that the random field exhibits realistic space and time scales. Using typical values obtained from our experience with the GENE code [27, 28] and renormalizing them to a temperature of $10 \mathrm{keV}$, we find a typical correlation length of $\lambda_{c}=1.6 \mathrm{~cm}$, a correlation time of $\tau_{c}=1.8 \cdot 10^{-4} \mathrm{~s}$, a mean $E \times B$ drift velocity of $V=900 \mathrm{~m} / \mathrm{s}$, as well as a diamagnetic drift velocity of $v_{\mathrm{dr}} \sim 300 \mathrm{~m} / \mathrm{s}$. However, the latter may vary strongly depending on the gradient drive. These parameters lead to a Kubo number of $K \equiv V \tau_{c} / \lambda_{c} \approx 10$, which is in line with nonlinear gyrokinetic simulations.[7] We note in passing, however, that the value of the Kubo number does not affect the key conclusions of the present work. Also, we will only consider fluctuations which are isotropic in the perpendicular plane $\left(\lambda_{x}=\lambda_{y}\right)$; geometrical effects due to streamer formation [29] are not treated. However, we will see that in the case of fast particles, they are of limited relevance, anyway.

\section{PARTICLE MOTION}

For simulating the particle trajectories, we use the full equations of motion preserving the Hamiltonian properties of the system:[30]

$$
\begin{aligned}
\frac{d \mathbf{X}}{d t} & =\mathbf{v}_{\|}+\frac{B}{B_{\|}^{*}}(\underbrace{\frac{\mu}{q B^{2}} \mathbf{B} \times \nabla B}_{v_{\nabla B}} \\
& +\underbrace{\frac{m v_{\|}^{2}}{q B^{3}}\left[\mathbf{B} \times \nabla B+B \nabla \times \mathbf{B}-\frac{\mathbf{B}}{B}(\mathbf{B}(\nabla \times \mathbf{B}))\right]}_{v_{\text {curv }}}-\frac{\nabla \phi^{\mathrm{eff}} \times \mathbf{B}}{B^{2}}), \\
\frac{d v_{\|}}{d t} & =\frac{1}{m v_{\|}} \frac{d \mathbf{X}}{d t} \cdot\left(-q \nabla \phi^{\mathrm{eff}}-\mu \nabla B\right) .
\end{aligned}
$$


Here, $\phi^{\text {eff }}$ denotes the gyroaveraged electrostatic potential and $B_{\|}^{*}=B+m v_{\|} /\left(e B^{2}\right)(\nabla \times \mathbf{B}) \cdot \mathbf{B}$. We note in passing that the correction term included in $B_{\|}^{*}$ is negligible in general. The particles are tracked by a modified version of the Gourdon code, [31] which uses an explicit Adams-Bashforth algorithm to integrate Eq. (7).

Depending on the pitch angle parameter $\eta=v_{\|} / v$, particles can circulate around the torus, following the magnetic field lines (passing orbits, large $\eta$ ), or they can be reflected by the mirror force and bounce between two poloidal reversal points (trapped orbits, small $\eta$ ). In both cases, the particles do not follow the magnetic lines exactly any more, but deviate in an oscillatory fashion in the $x$ as well as in the $y$ direction due to the curvature and $\nabla B$ drifts. In addition to that, the particles drift in the $y$ direction with a constant velocity (toroidal precession). Fig. 1 shows two examples of fast particle orbits in front of the background potential. In field-aligned coordinates, the orbit motion can be described as a superposition of a circular (elliptical) motion in the $x-y$ plane and a constant drift motion in the $y$ direction. An analytical treatment of the orbit motion in the $R-z$ plane can be found in Ref. [16], and a simple expression for the $y$ drift in the small- $\epsilon$ limit is given by $v_{y}(z)=\left(v_{\nabla B}+v_{\text {curv }}\right) h(z)$ (see, e.g., Ref. [9]) where $h(z) \equiv \cos z+\hat{s} z \sin z$ and $\hat{s}(r)=r / q(r) d q(r) / d r$. Since we are interested in the average value over one orbit turn, we define $\bar{h}=1 /\left(2 z_{0}\right) \int_{-z_{0}}^{z_{0}} h(z) d z$. This yields $\bar{h}=\hat{s}$ for passing particles $\left(z_{0}=\pi\right)$ and $\bar{h} \rightarrow 1$ for $z_{0} \rightarrow 0$ for deeply trapped particles. Since the particles do not move along $z$ with constant velocity, such an approximation is rather crude, though. Moreover, we have $\epsilon \approx 0.1$, such that one may expect finite aspect ratio corrections to play a role. Employing, nevertheless, this simple model, the orbit parameters in the case of passing particles are given by

$$
T_{\text {orbit }} \approx \frac{2 \pi q_{0} R_{0}}{\eta} \sqrt{\frac{m}{2 E}}, \quad \Delta x \approx \frac{2 \eta q_{0}}{e B_{0}} \sqrt{2 m E}, \quad v_{y} \approx \frac{2 \eta^{2} \hat{s}}{e B_{0} R_{0}} E .
$$

For our nominal parameters, these expressions read

$$
\begin{array}{r}
T_{\text {orbit }} / s \approx 1.3 \cdot 10^{-4} q_{0} / \sqrt{E / \mathrm{keV}}, \quad \Delta x / m \approx 0.0025 q_{0} \sqrt{E / \mathrm{keV}} \\
v_{y} /(\mathrm{m} / \mathrm{s}) \approx 60 \hat{s} E / \mathrm{keV}
\end{array}
$$

For trapped particles, on the other hand, the orbit parameters are given by

$$
T_{\text {orbit }} \approx 2 \pi q_{0} R_{0} \sqrt{\frac{m}{\left(1-\eta^{2}\right) \epsilon E}}, \quad \Delta x \approx \frac{2 \eta q_{0}}{e B_{0} \epsilon} \sqrt{2 m E}, \quad v_{y} \approx \frac{(1-\eta)^{2}}{e B_{0} R_{0}} E .
$$

For our nominal parameters, this is

$$
\begin{array}{r}
T_{\text {orbit }} / s \approx 5.4 \cdot 10^{-4} q_{0} / \sqrt{E / \mathrm{keV}}, \quad \Delta x / m \approx 0.003\left(q_{0} / r_{0}\right) \sqrt{E / \mathrm{keV}} \\
v_{y} /(\mathrm{m} / \mathrm{s}) \approx 29 E / \mathrm{keV}
\end{array}
$$

The simulation results presented in this paper show that the expressions for $T_{\text {orbit }}$ and $\Delta x$ fit almost perfectly, whereas the expression for $v_{y}$ is correct only within about $30 \%$, which may be attibuted to finite aspect ratio effects and/or to our simple approach of averaging over $z$. Later, we present these parameters as simulation results in tables, so that the reader can check the validity of the above expressions on his own. We are not aware of an analytical expression for the diameter of the elliptical motion in the $y$ direction. However, the simulations show that it coincides with the radial diameter up to an error of at most a few 10\%. Consequently, in the framework of simple analytical descriptions, it seems reasonable to assume $\Delta y \approx \Delta x$.

The particle motion illustrated in Fig. 1 has already been the subject of study in Ref. [7], where the interaction of gyrating particles with a background turbulence drifting in the $y$ direction has been investigated and explained. The present case with particles circulating on drift orbits and drifting with respect to the background potential is essentially the same. In the former work, it was shown that an electrostatic potential drifting with a velocity $v_{\mathrm{dr}}$ in the $y$ direction strongly reduces transport in the $x$ direction above a so-called "drop time" $\tau_{\text {drop }}=2 \lambda_{c} / v_{\mathrm{dr}}$, since, in a frame of reference moving with the drift velocity, Lorentz invariance (in the non-relativistic limit) leads to an additional electric field, acting as a transport barrier. $\tau_{\text {drop }}$ is the mean time a particle needs to run against the barrier and being reflected. So for $\tau_{\text {drop }}<\tau_{c}$, a significant transport reduction can be expected, whereas in the opposite case, no significant drift-induced effect is observable, since the barrier does not exist long enough for the particle to feel its presence. The gyroaveraging procedure and its influence on the transport level has also been described before.[6, 7]

Now, it is not only the particles which are subject to a drift, but also the background potential. Depending on the temperature and density gradients of the plasma, this drift can have velocities of up to about $5 \rho_{s} c_{s} / R_{0}$, [9] which may be as large as several km/s for our nominal parameters. In Ref. [9], the effect of a resonance between the diamagnetic drift of the background potential and the curvature drift of beam ions has already been described. In a number of simulations we found that the resonance width (i.e., the relative drift velocity for which the diffusivity drops to $1 / e$ 
of the original value) can be expressed as $\Delta_{1 / e} v_{\mathrm{d} r}=2 \lambda_{c, y} / \tau_{c}$. This simply means that the diffusivity is reduced by

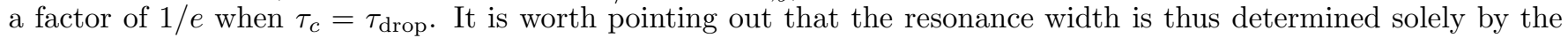
turbulence properties $\lambda_{c}$ and $\tau_{c}$. In the following, we will denote the drift of the background potential by $v_{\mathrm{dr}}$, whereas the test particle drifts will be denoted by $v_{y}$.

\section{FUNDAMENTAL TRANSPORT AND DECORRELATION MECHANISMS}

\section{A. Orbit averaging}

The gyromotion of a particle can be treated by the well-known "gyroaveraging" procedure (see, e.g., Ref. [14]). Here, the electrostatic potential is replaced by a new effective potential,

$$
\phi^{\mathrm{eff}}(\mathbf{x}) \equiv(2 \pi)^{-2} \int_{-\infty}^{\infty} d \mathbf{k} e^{i \mathbf{k} \cdot \mathbf{x}} \phi(\mathbf{k}) J_{0}\left(k \rho_{g}\right)
$$

where $J_{0}$ is the Bessel function of order zero. The gyroaveraged drift velocity $V^{\mathrm{e} f f}$ can then be derived from this effective potential. Furthermore, the correlation length of the gyroaveraged potential changes. For $\rho_{g} \gtrsim \lambda_{c}$, the effective values can be approximated by [6]

$$
V^{\mathrm{eff}}=V\left(4 \sqrt{\pi} \rho_{g} / \lambda_{c}\right)^{-1 / 2}, \quad \lambda_{c}^{\mathrm{eff}}=1.73 \lambda_{c} .
$$

Now, recalling the discussion concerning Fig. 1, we may expect that a similar procedure is possible with respect to the orbit motion of a particle, since, in principle, we just have to replace $\rho_{g}$ with $\Delta x / 2$ and $v_{\mathrm{dr}}$ with $v_{y}-v_{\mathrm{dr}}$. However, we have to remember that the orbit motion takes place on much slower time scales than the gyromotion. So, in a first step, we want to clarify the condition under which orbit averaging is valid.

In Fig. 2, the orbit motion of a particle with a rather large $E \times B$ drift velocity is plotted in front of a fluctuating potential symbolized by the contours of its autocorrelation function. As explained in the caption, a criterion for the validity of orbit averaging is the condition $T_{\text {orbit }} \bar{V}^{\text {eff }}<\lambda_{c}, T_{\text {orbit }}$ being the cycle duration. Additionally to the $E \times B$ drift, decorrelation can be caused by the curvature drift velocity relative to the diamagnetic drift velocity of the background potential. Moreover, the correlation time of the fluctuations must, of course, be large compared to the cycle duration, $T_{\text {orbit }} \ll \tau_{c}$. Consequently, we can state as a necessary condition for the validity of orbit averaging the relations

$$
\Xi_{\text {o.a. }} \equiv \max \left\{\bar{V}^{\text {eff }},\left|v_{\text {dr }}-v_{y}\right|\right\} \frac{T_{\text {orbit }}}{\lambda_{c}}<1, \quad T_{\text {orbit }} \ll \tau_{c} .
$$

If one of those inequalities is violated, the particle is decorrelated before it completes one turn, and therefore, orbit averaging is not applicable anymore. If orbit averaging is valid, the gyroradius $\rho_{g}$ in Eq. (12) can simply be replaced by half of the deviation of the particle from the original flux surface, $\Delta x / 2$ (where we may assume that $\Delta y \approx \Delta x$ ). As we will see, the first relation of Eq. (14) is very critical, and the transport level for fast particles strongly depends on its validity. Our numerical simulations will show that for orbit averaging to be valid, the introduced parameter actually has to be somewhat smaller than unity for most cases. From now on we want to use the notation $V^{\text {eff }}$ and $\phi^{\text {eff }}$ for gyroaveraged values, whereas we write $\bar{V}^{\text {eff }}$ and $\bar{\phi}^{\text {eff }}$ for orbitaveraged variables.

\section{B. Decorrelation mechanisms}

We now want to analyze different decorrelation mechanisms for the motion of fast particles in a tokamak. As long as a Lagrangian correlation between the current particle velocity and the velocity at its starting position exists, the transport is, in general, not diffusive. For example, for small times, before a particle feels the structure or the time dependence of the turbulent streamfunction, it moves ballistically, inducing superdiffusive transport. This is the case for $t<\tau_{\mathrm{ff}} \equiv \lambda_{c} / V, \tau_{\mathrm{ff}}$ being the average time of flight of a particle exploring a typical fluctuation structure. On the other hand, as long as a certain structure persists, the particle can get trapped in it, or be bound by a transport barrier produced by the diamagnetic drift of the background potential. In this case, the transport will be subdiffusive. Only when the particle starts to forget the information concerning its starting point, i.e., when there is no more correlation between the streamfunction at the current position and at the starting position of the particle, its motion becomes diffusive. This may be seen from the Taylor relation[32]

$$
D_{x}(t) \equiv \frac{1}{2} \frac{d}{d t}\left\langle x(t)^{2}\right\rangle=\int_{0}^{t} d \tau L_{v_{x}}(\tau)
$$


where $D_{x}$ is the diffusion coefficient along the $x$ axis, $L_{v_{x}}$ is the Lagrangian autocorrelation function of the respective particle velocities, and the angular brackets denote ensemble averaging. When $L_{v_{x}}(t)$ becomes zero, $D_{x}(t)$ becomes constant.

In two dimensions, the only relevant decorrelation mechanism is the time dependence of the electrostatic potential. This means that the effective decorrelation time of the particle motion is equal to the correlation time of the potential, $\tau^{\text {eff }} \equiv \tau_{c}$. [15] If there is no time dependence at all, the particles simply follow the equipotential lines of the electrostatic potential (the streamfunction); thus, their dynamics is fully deterministic. In a simple approach, diffusion coefficients can be obtained by following the $D(t)$ curve for a static potential, while the curve is forced to saturate at $t=\tau_{c}$. If there is a background drift, a sharp drop of the curve occurs at $t=\tau_{\text {drop }}$ if $\tau_{\text {drop }}<\tau_{c}$. [7] Fig. 3 illustrates this type of behavior in two dimensions.

Now, in three dimensions, the situation is basically the same as long as orbit averaging is valid. In this case, the only influence of the orbit motion is the averaging of the background potential which reduces the transport for $\Delta x / 2>\lambda_{c}$ (in addition to the reduction produced by averaging over the gyroorbit). The situation is quite different, however, if orbit averaging is not valid. In that case, as we have seen while discussing Fig. 2, the decorrelation is produced by the orbit motion of the particle. So, as an effective decorrelation time, it is reasonable to take the time a particle needs to cross a distance of one correlation length during its orbit motion, i.e.,

$$
\tau^{\text {orbit }}=\lambda_{c} / v_{\text {orbit }}=\lambda_{c} T_{\text {orbit }} /(\pi \Delta x) \text {. }
$$

However, this relation is only valid if $\Delta x / 2 \gtrsim \lambda_{c}$. Only then is the drift orbit wide enough to produce decorrelation. In the opposite case, if $\Delta x / 2 \lesssim \lambda_{c}$, the situation is more complicated. Here, the particle does not necessarily decorrelate at $t=\tau^{\text {orbit }}$, since the orbit radius is smaller than the correlation length; and even if orbit averaging is not valid, the $E \times B$ motion may force the particle to follow the equipotential lines of the streamfunction. So although it travels a distance larger than $\lambda_{c}$ during one orbit turn, it does not necessarily decorrelate. On the other hand, since orbit averaging is not valid, the particle is not strictly bound to the equipotential lines. So, in this case, only a range of time scales can be given at which decorrelation occurs, namely $\tau_{\mathrm{fl}} \leq \tau^{\text {eff }} \leq \tau_{c}$.

As an additional decorrelation mechanism in three dimensions, one obtains the parallel motion of the particles. The relevant time scale is given by $\lambda_{\|} / v_{\|}$where $\lambda_{\|}$is the parallel correlation length of the fluctuations.

In summary, we thus have:

$$
\begin{aligned}
& \tau^{\text {eff }}=\min \left\{\tau_{c}, \frac{\lambda_{\|}}{v_{\|}}\right\} \quad \forall \quad \Xi_{\text {o. } a .} \lesssim 1 \\
& \tau^{\text {eff }}=\min \left\{\tau_{c}, \frac{\lambda_{\|}}{v_{\|}}, \quad \tau^{\text {orbit }}=\lambda_{c} T_{\text {orbit }} /(\pi \Delta x)\right\} \quad \forall \quad \Xi_{\text {o. } a . ~} \gtrsim 1
\end{aligned}
$$

Here, $\tau^{\text {eff }}$ is the effective decorrelation time for which the $D(t)$ curve departs from a reference curve without 3D effects and saturates. For the saturation level, it remains crucial whether the effective decorrelation time is larger

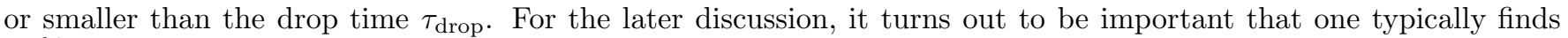
$\tau^{\text {orbit }}<\tau_{\text {drop }}<\tau_{c}$, which means that the drift barrier induced by the magnetic drifts of the particles and the diamagnetic drift of the background potential exists if $\Xi_{\text {o.a. }}<1$, but not if $\Xi_{\text {o.a. }}>1$.

\section{A simple 2D model}

To gain a better understanding of the mechanisms we have just explained, it is useful to study the drift orbit motion in the framework of a simple 2D model defined by:

$$
\dot{\mathbf{x}}=\mathbf{v}-\frac{1}{B} \nabla \phi^{\mathrm{eff}} \times \mathbf{e}_{z}, \quad \dot{\mathbf{v}}=\omega_{\text {orbit }} \mathbf{v} \times \mathbf{e}_{z} .
$$

Eq. (18) describes a particle which is forced on an orbit with $\omega_{\text {orbit }}=2 \pi / T_{\text {orbit }}$, at the same time undergoing $E \times B$ drift motion. The orbit radius is set by choosing an appropriate starting velocity of the particle, $v(t=0)=\Delta x \omega_{\mathrm{orbit}} / 2$. In this $2 \mathrm{D}$ system, we can also perform orbit averaging of the (already gyroaveraged) potential $\phi^{\text {eff }}$ over a circular orbit with radius $\Delta x / 2$. Simulation results based on this model will be presented below.

\section{BEAM IONS IN A SHEARLESS MAGNETIC FIELD}

Let us now turn to $3 \mathrm{D}$ simulations. In a first step, we consider a magnetic field with $q(r) \equiv 1.4, \hat{s}(r) \equiv 0$. Here, the magnetic field lines close after 5 poloidal ( 7 toroidal) turns, and therefore it is possible to choose $\lambda_{\|}=\infty$ which makes 
comparisons with the simplified model easier. To ensure continuity in the $z$ direction, the flux surface is covered by $M=5$ identical copies of a flux tube. Moreover, we will see that this special case exhibits many features which are typical of more general weak-shear situations. For the 3D particle simulations with the Gourdon code, we consider deuterium ions with $\eta=0.99$ and energies ranging from $10 \mathrm{keV}$ to $160 \mathrm{keV}$ which we insert at $r_{0}=0.7$ at the outboard midplane $(\theta=0)$. The characteristic orbit parameters can be found in Table I. For comparison, we then recompute these orbits using the simple 2D model of Eq. (18), both for the forced drift orbit case as well as for the orbit averaged case. In addition, we do these simulations both with and without a background drift of $v_{\mathrm{dr}}=300 \mathrm{~m} / \mathrm{s}$. Fig. $4 \mathrm{shows}$ the saturated values of the diffusion coefficients for the described cases. We see that the 3D curves, Eq. (7), and the 2D curves, Eq. (18), agree with each other within a factor of about 1.5, whereas the orbit averaged curves deviate by a larger factor. This behavior can be understood by inspecting the orbit parameters shown in Table I. For the curves without a background drift (black lines), the orbit averaged curves exhibit a rather fast drop for energies exceeding 40 $\mathrm{keV}$, which is due to the fact that $\tau_{\text {drop }}<\tau_{c}$. The orbit averaged curve with the background drift has its maximum at $E=40 \mathrm{keV}$, since there the drift velocity of the particle is in resonance with the diamagnetic drift velocity, and therefore $\tau_{\text {drop }} \gg \tau_{c}$. As $\Xi_{\text {o.a. }}$. is close to unity for all curves, there are deviations between the orbit averaged and the exact curves, as could be expected. However, the shape of the curves is similar. The correspondence works best between $E=40 \mathrm{keV}$ and $80 \mathrm{keV}$ since for these cases, $\Xi_{\text {o. } a .} \ll 1$. Given that the transport is governed by the validity of the orbit averaging approach, one then finds a rather fast decline of $D(E)$ towards higher energies.

More insight into the mechanisms at work can be gained from Fig. 5. Here, the running (i.e., time dependent) diffusion coefficient is plotted both for the full $2 \mathrm{D}$ dynamics and the respective orbit averaged case for $v_{\mathrm{dr}}=0$. We first concentrate on the orbit averaged curves. Here, one observes that for small energies (i.e, small curvature drift velocities), the diffusion coefficient first increases linearly in time (ballistic regime), then it decreases slightly (trapping effects), and finally it saturates (decorrelation due to $t>\tau_{c}$ ). For larger energies (i.e., larger curvature drift velocities), one has $\tau_{\text {drop }}<\tau_{c}$, and one observes a strong decrease due to the existence of a "drift barrier." In the ballistic regime, we observe the reduction of the $E \times B$ drift velocity due to the orbit averaging, $D \sim\left(\bar{V}^{\text {eff }}\right)^{2} t$. The curves obtained for the exact $2 \mathrm{D}$ orbit motion are similar, but the underlying mechanisms are completely different. For $t<T_{\text {orbit }}$, orbit averaging is not applicable yet. Therefore, it is the original $E \times B$ drift which causes the diffusion of the particles, and for small times, all the curves follow the ballistic diffusion equation, $D=V^{2} t$. For larger times, there

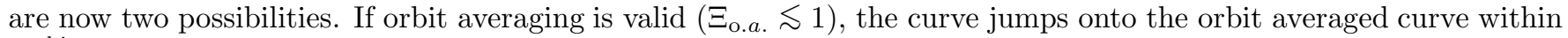
$\tau^{\text {orbit }} \lesssim t \lesssim T_{\text {orbit. }}$. This relation describes the time span during which the particle already feels the stochasticity, but orbit averaging is not valid yet. As can be seen from Fig. 5 as well as from Table I, this mechanism applies for $E=40$ $\mathrm{keV}$ to $E=160 \mathrm{keV}$, but the saturation values of the curves are not identical since the validity of orbit averaging seems to be not fully established yet. The second possibility is that orbit averaging is clearly invalid $\left(\Xi_{\text {o.a. }}>1\right)$. In that case, $D(t)$ simply saturates at $t \sim \tau^{\text {orbit }}$, since, after having crossing the correlated zone once, it never returns to a correlated region. We observe this kind of behavior for small particle energies. For the case with a background potential drift, we obtain similar $D(t)$ curves.

In order to further clarify the decorrelation mechanisms at work, we consider a more idealized situation. In Fig. 6, the running diffusion coefficients are plotted for a number of $2 \mathrm{D}$ test particles with no drift and the same orbit width, differing only by their orbit circulation time $T_{\text {orbit }}$, which, in turn, determines the parameter $\Xi_{\text {o.a. }}$. For $T_{\text {orbit }} \leq 5.6 \cdot 10^{-5}$, we have $\Xi_{\text {o.a. }}<1$, and the curves approach the orbit averaged curve between $\tau^{\text {orbit }}<t<T_{\text {orbit }}$. For $T_{\text {orbit }}>5.6 \cdot 10^{-5}$, orbit averaging is not valid; hence, there is no trend to approach the orbit averaged curve, the diffusion coefficient follows the curve without any orbit effects, until it saturates at $t \sim \tau^{\text {orbit }}$, or, if $\tau^{\text {orbit }}>\tau_{c}$, at $t=\tau_{c}\left(=1.8 \cdot 10^{-4} s\right)$. Since in the ballistic regime, $D(t)=\left(\bar{V}^{\text {eff }}\right)^{2} t$ for the orbit averaging case, but $D(t)=V^{2} t$ in the case that orbit averaging is not valid (yet), the saturation levels for $D$ are higher in the latter case. So from this figure, it becomes clear how the fact that orbit averaging is not valid can lead to an increase of diffusivity. Also, one can predict that in that case, a reduction of the diffusivity can only occur if the effective decorrelation time $\tau^{\text {eff }}=\tau^{\text {orbit }}=\lambda_{c} T_{\text {orbit }} /(\pi \Delta x)$ is small enough to be in the ballistic regime where a reduction of $\tau^{\text {eff }}$ means a reduction of $D$. Since $\Delta x \propto E^{1 / 2}$ and $T_{\text {orbit }} \propto E^{-1 / 2}$, we therefore expect a decrease like $D \propto E^{-1}$ for large energies. More precisely, since the maximum of the static $D(t)$ curve lies at $t=\tau_{\mathrm{fl}}=\lambda_{c} / V$, the criterion for the $E^{-1}$ decrease is $\tau^{\text {eff }}<\tau_{\text {fl }}$, i.e.,

$$
\frac{T_{\text {orbit }} V}{\pi \Delta x}<1
$$

Inserting the equations for $T_{\text {orbit }}$ and $\Delta x$ for our nominal physical parameters and for passing orbits into Eq. (19), we obtain the condition $E \gtrsim 15 \mathrm{keV}$ for the $1 / E$ decrease. However, we have to remember that this criterion is valid only if orbit averaging is invalid, and if $\tau_{\text {drop }}>\tau^{\text {orbit }}$. Moreover, for the $T_{\text {orbit }}=5.6 \cdot 10^{-6}$ curve, the bursty nature of the diffusion can be seen. A burst occurs every time the particles come back into their original zone of correlation, i.e., after $\Delta t=T_{\text {orbit }}$. 


\section{BEAM IONS IN A SHEARED MAGNETIC FIELD}

\section{A. Simulation results}

We now turn to a more realistic scenario by employing a sheared magnetic field with $q(r)=0.5 r^{2}+1.25$. At the same time, we choose the envelope in Eq. (6) to be $g(z)=0.5 \cos (z-\sin (z))+0.5$. This ansatz reproduces the typical ballooning structure of plasma turbulence and implies a parallel correlation length of $\lambda_{\|} \sim 2 \pi q_{0} R_{0}$ which is in agreement with typical experimental results.[33-36] The beam ion and turbulence parameters are the same as before, and the beam energies are varied from $E=10 \mathrm{keV}$ to $E=1280 \mathrm{keV}$. Because of the magnetic shear, the curvature drift velocities $v_{y}$ are much higher now than in the shearless case. All simulations are performed for a diamagnetic drift velocity of the background fluctuations of either $v_{\mathrm{dr}}=0$ or $v_{\mathrm{dr}}=1 \mathrm{~km} / \mathrm{s}$. The characteristic orbit parameters can be found in Table II. The saturated diffusion coefficients (as a function of the beam energies) for the 3D motion, the corresponding 2D orbit motion results, and the orbit averaged 2D cases are shown in Fig. 7. For the moment, we want to ignore the dashed bold lines. As can be inferred from Table II, the drift velocities are higher than in the shearless case, leading to an increase of $\Xi_{\text {o.a. }}$. Consequently, the condition $\Xi_{\text {o.a. }}<1$ is only fulfilled for a single case, namely $E=40 \mathrm{keV}$ and $v_{\mathrm{dr}}=1 \mathrm{~km} / \mathrm{s}\left(v_{y} \approx v_{\mathrm{dr}}\right)$. In Fig. 7 , we see that only for these parameters, the orbit averaged value corresponds to the orbit motion value. Moreover, we observe that the $2 \mathrm{D}$ orbit curves correspond quite well to the $3 \mathrm{D}$ curves, except for $E \sim 100 \mathrm{keV}$ and $v_{\mathrm{dr}}=1 \mathrm{~km} / \mathrm{s}$. This may be attributed to the fact that $\Xi_{\text {o.a. }}$ is close to unity in that case, which means that the fast drop in the orbit averaged curves still affects the $2 \mathrm{D}$ orbit curves. However, we see that $T_{\text {orbit }} \lesssim \tau_{\text {drop }}$, and in three dimensions, the particle decorrelates at $t \sim T_{\text {orbit }}$ because of its parallel motion. Therefore, it is not affected by the drift barrier. In all the cases where orbit averaging is clearly not valid, decorrelation is produced by $\tau^{\text {eff }}=\tau^{\text {orbit }}=\lambda_{c} T_{\text {orbit }} /(\pi \Delta x)$, which is always smaller than $T_{\text {orbit }}$. Therefore, parallel decorrelation effects are irrelevant in that case, and the $3 \mathrm{D}$ motion corresponds to the $2 \mathrm{D}$ motion. We note in passing that this would still be valid if the parallel correlation length would be reduced by up to one order of magnitude. Especially for larger beam energies, we observe a very clear $1 / E$ decrease which we have already explained in the previous section. Only in the case of the drifting background potential, larger deviations occur because of the existence of a drift resonance around $40 \mathrm{keV}$.

Based on these insights, the value of $D$ can be estimated. Since the decorrelation due to the orbit motion occurs within the ballistic regime $\tau^{\text {orbit }} \ll \tau_{\text {fl }}$, we get $D \sim V^{2} \tau^{\text {eff }}$ (low Kubo number limit). For our nominal parameters, this means $D \sim 228 \mathrm{~m}^{2} / \mathrm{s}(E / \mathrm{keV})^{-1}$, which is correct within a factor of three. This deviation can be taken into account assuming that the decorrelation occurs not exactly at $\tau^{\text {eff }}$ but already at about $\tau^{\text {eff }} / 3$. Including this correction factor and generalizing to arbitrary physical parameters, we find the relation

$$
D \approx \frac{V^{2} \lambda_{c} R_{0} B_{0} e}{6 \eta^{2} E} \approx \frac{\left\langle\phi^{2}\right\rangle R_{0} e}{6 \lambda_{c} B_{0} \eta^{2} E}
$$

for beam ions in electrostatic tokamak microturbulence for $\Xi_{\text {o.a. }}>1$.

Using some normalizations that are common in simulations of plasma turbulence, it is possible to rescale Eq. (20) to arbitrary temperatures and machine sizes. To this aim, we use $\lambda_{c, \operatorname{dim} . \text { less }}=\lambda_{c, \mathrm{SI}} / \rho_{s}$ and $\phi_{\operatorname{dim} . \text { less }}=\phi_{\mathrm{SI}} e L_{\perp} /\left(k_{B} T_{e} \rho_{s}\right)$, with $\rho_{s} \equiv c_{s} m /\left(e B_{0}\right)$, and $c_{s}=\sqrt{k_{B} T_{e} / m}$ being the ion sound speed.[28] Inserting these relations into Eq. (20), we obtain

$$
D \approx \frac{\left\langle\phi_{\text {dim.less }}^{2}\right\rangle R_{0} m^{0.5}\left(k_{B} T_{e}\right)^{2.5}}{6 \lambda_{\text {dim.less }} e^{2} B_{0}^{2} \eta^{2} L_{\perp}^{2} E} \sim \frac{m^{0.5}\left(k_{B} T_{e}\right)^{2.5}}{e^{2} B_{0}^{2} R_{0} E}=\left(\frac{E}{k_{B} T_{e}}\right)^{-1} D_{\text {gyroBohm }}
$$

This is the well known gyro-Bohm diffusion coefficient, $D_{\text {gyroBohm }}=\rho_{s}^{2} c_{s} / R_{0}$, multiplied with the inverse of the particle energy normalized with respect to the thermal energy of the background plasma. In this context, we would like to point out that the occurrence of the ratio $E / T_{e}$ is not trivial since the thermal energy determines the scales of the background turbulence while the particle energy governs the orbit motion of the energetic particles. Both influence the fast particle transport, but they are a priori independent. To clarify this point, let us give a counter-example. Assuming that the decorrelation is not caused by the perpendicular orbit motion but by the parallel motion, then the effective decorrelation time would be $\tau^{\text {eff }}=T_{\text {orbit }} \propto E^{-1 / 2}$ instead of $\tau^{\text {eff }} \propto E^{-1}$. This, in consequence, would

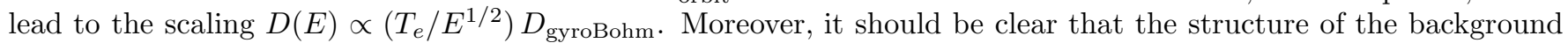
turbulence (e.g. streamers) is of minor relevance for the transport of fast particles if decorrelation is caused by the orbit motion, since $\tau^{\text {orbit }}<\tau_{\mathrm{fl}}$.

In closing this section, we briefly want to compare the results of Fig. 7 with those of previous works. In Ref. [9], a $1 / E$ decay of the diffusion coefficient was found for large particle energies in nonlinear gyrokinetic simulations, whereas an approximate $1 / E^{2.6}$ decay was found in quasilinear runs based on generalized Maxwellian distributions. 
In Ref. [10], quasilinear calculations for slowing-down distributions lead to a $1 / E^{3}$ decay. Comparing those results with each other as well as with ours, we may conclude that it is indeed the turbulent (i.e., random) nature of the advecting field which is responsible for the slow decay of the particle transport with increasing energy. A more rapid decay (faster than $1 / E$ ) is only obtained in cases for which the particle is not decorrelated after one orbit turn. This applies if orbit averging is valid $\left(\Xi_{\text {o.a. }} \lesssim 1\right)$, as we have already seen. In linear simulations, orbit decorrelation does not occur. Hence, orbit averaging remains valid and the reduction of $D$ with $E$ is overestimated.

It is instructive to generalize the expression for the validity of orbit averaging, Eq. (14), to arbitrary machine sizes and background temperatures as we have just done for the diffusion coefficient. For very large particle energies, we have $v_{y} \gg\left\{v_{\mathrm{dr}}, \bar{V}^{\mathrm{eff}}\right\}$, and therefore $\Xi_{\mathrm{o} . a .}=v_{y} T_{\mathrm{orbit}} / \lambda_{c}$. Inserting the respective terms into that equation, we find

$$
\Xi_{\text {o.a. }}=\frac{2^{1.5} \pi \eta \hat{s} q E^{0.5}}{\lambda_{\text {dim.less }}\left(k_{B} T_{e}\right)^{0.5}} \sim \hat{s} q\left(\frac{E}{k_{B} T_{e}}\right)^{0.5} .
$$

Interestingly, this expression is independent of $R_{0}$ and $B_{0}$. Thus, a reduction of the fast particle transport through a reduction of $\Xi_{\text {o.a. }}$. can be achieved, e.g., through a reduction of the safety factor or the magnetic shear. This result explains, on general grounds, why one can expect orbit averaging to apply and the fast ion transport to drop quickly with increasing particle energy in low-shear regions of a tokamak. Thus, the latter can, in fact, act as kind of "transport barriers" for energetic particles.

\section{B. Some comments on reduced-volume simulations}

At this point, it seems worthwhile to briefly discuss an unphysical aliasing-type effect which can occur if, in energetic particle studies, a flux surface is covered, for convenience, by $M$ identical copies of a thin flux tube, and $M$ is chosen too large. Our standard simulations are performed with $M=1$, i.e., the box width in the $y$ direction is chosen to be $2 \pi r_{0} / q_{0}$, and the particles only feel the true periodicity of the flux surface. Now, to reduce the computational effort in setting up the test potential, one might want to use $M>1$ instead. Here, one has to be careful, however, as will become clear presently. The dashed lines in Fig. 7 have been obtained by using $M=10$ instead of $M=1$, corresponding to a box width in the $y$ direction of $L_{y} \equiv 2 \pi r_{0} /\left(10 q_{0}\right) \approx 0.31 \mathrm{~m}$. For thermal particle velocities, such a choice would be fully adequate, since the time a particle needs to cross the box is much larger than the turbulence correlation time $\tau_{c}$. Hence, the particle encounters a new realization when reentering the simulation volume. Now, for $M=10$, the minimum velocity a particle needs to have to feel the periodicity is $v_{\min }=L_{y} / \tau_{c} \approx 1750 \mathrm{~m} / \mathrm{s}($ for $\left.r_{0}=0.7 \mathrm{~m}\right)$. From Table II, we can infer that the critical velocity is reached at $E \sim 80 \mathrm{keV}$, and from Fig. 7 , we see that this is indeed (at least approximately) the particle energy at which the curves begin to deviate (in fact, since we have only simulated a discrete number of energies, $E=160 \mathrm{keV}$ is the first one where we observe the difference).

In Fig. 8, the mechanism is explained which leads to an unphysical decrease of the diffusivity for $M=10$. It displays a trajectory subject to a large drift velocity which takes the particle out of the correlated zone (see also Fig. 2). However, because of the imposed periodicity, the particle gets back into the zone of correlation when it reenters the simulation volume. So after one turn, the particle, although having traveled a distance of $T_{\text {orbit }} v_{y}$, feels a potential which corresponds to the much smaller distance $T_{\text {orbit }} v_{y}^{\text {eff }}$, where $v_{y}^{\text {eff }}=\left(T_{\text {orbit }} v_{y}^{\text {eff }} \bmod L_{y}\right) / T_{\text {orbit }}$. For the transport, this means that the decorrelation does not occur at $\tau^{\text {orbit }}=\lambda_{c} / v_{\text {orbit }}$ anymore. Since $\Xi_{\text {o.a. }}=T_{\text {orbit }} v_{y}^{\text {eff }} / \lambda_{c}<1$, orbit averaging applies again, and, if $\tau_{\text {drop }}<\min \left\{T_{\text {orbit }}, \tau_{c}\right\}$, the drift barrier becomes dominant and the transport is reduced. It should be clear that such a re-correlation can only occur if the characteristic time scales are smaller than the correlation time $\tau_{c}$ of the potential. This is true in our case. So, in general, if performing fast particle simulations with $M>1$, one should always ensure that $M$ is small enough for such aliasing effects not to occur.

\section{Breaking of adiabatic invariants}

Our above findings can also be looked at from a more abstract point of view. As is well known, the radial transport of both thermal and suprathermal particles is fundamentally connected with the breaking of certain adiabatic invariants. In $3 \mathrm{D}$, the phase space can be parameterized by $\mathbf{J}=\left(\mu, J_{\phi}, J_{p}\right)$ and $\theta=\left(\theta_{g}, \theta_{\phi}, \theta_{p}\right)$, where $\mu$ is the magnetic moment, $J_{\phi}$ is the canonical angular momentum, and $J_{p}$ is the poloidal flux enclosed by the drift surface.[37, 38] The vector $\theta$ contains the corresponding phases (the canonical conjugate values to $\mathbf{J}$ ), and $\omega_{g}=\dot{\theta}_{g}, \omega_{\phi}=\dot{\theta}_{\phi}$, and $\omega_{p}=\dot{\theta}_{p}$ are the frequencies of the periodic motions. Diffusion in real space corresponds to a diffusion in $\mathbf{J}$ space which presupposes a breaking of one or more of the adiabatic invariants $\mu, J_{\phi}$, and $J_{p}$. This may be caused by resonances between the frequencies associated with the respective periodic motions and the perturbation frequencies $\omega_{\text {turb }}$ of the background turbulence. The resonance condition can be expressed as $\omega_{\text {turb }}=l_{g} \omega_{g}+l_{\phi} \omega_{\phi}+l_{p} \omega_{p},\left(l_{g}, l_{\phi}, l_{p}\right.$ 
integer).[37, 38] While $\omega_{g}$ will always be much too large to be in resonance with the turbulence and, for fast ions, $\omega_{\text {turb }} / \omega_{\phi} \sim\left(k_{y} \rho_{s}\right)\left(c_{s} / R_{0}\right)\left(q_{0} R_{0} / v_{\|}\right) \ll 1$, the frequency corresponding to the third invariant is often found to be comparable to or smaller than the typical frequencies of the fluctuations.[17] Since $\omega_{p}$ is the frequency with which the particles drift around the torus in the toroidal direction, it is given by $\omega_{p}=v_{y} / r_{0}$. Our test potential was created using a Gaussian frequency spectrum with an e-folding frequency of about $167001 / \mathrm{s}$. This frequency is only reached by $\omega_{p}$ at $E \sim 500 \mathrm{keV}$. Therefore, a significant radial transport of fast particles with $E \lesssim 500 \mathrm{keV}$ is possible due to the breaking of the third adiabatic invariant by the background turbulence.

\section{TRAPPED IONS IN A SHEARED MAGNETIC FIELD}

Up to now, we have concentrated on beam-like ions, characterized by $\eta \sim 1$. This was, in part, motivated by recent experimental results [11] concerning the efficiency of neutral beam injection, and the need to explain them. In the present section, however, we would like to investigate in which way the $D(E)$ behavior is modified if the test particles only have a small parallel velocity component such that they are trapped on the outer side of the torus, moving along banana orbits. Taking $\eta=0.2$, the respective orbit parameters are shown in Table III. Comparing them with the ones in Table II, one sees that the orbit circulation time is slower than for the passing particles with $\eta=0.99$, as could be expected. Therefore, $\Xi_{\text {o.a. }}$ is larger, and orbit averaging cannot be applied. Hence, the decorrelation is expected to be caused by $\tau^{\text {orbit }}$ in all cases. On the other hand, since the particles now have a significant perpendicular velocity component, finite gyroradius effects have to be taken into account. As can be inferred from the table, $\rho_{g}>\lambda_{c}$ for $E>160 \mathrm{keV}$. Since $\tau^{\text {orbit }}$ is larger than for the $\eta=0.99$ case, we expect the base level of $D(E)$ to be larger, too. However, due to gyroaveraging, the effective $E \times B$ drift velocity is reduced for larger particle energies, leading to a faster drop of the diffusivity. In Fig. 9, several $D(E)$ curves are plotted. It can be seen that the fall-off of the diffusivity with growing particle energy is clearly faster than for the beam ion case. It is interesting to compare the $D(E)$ curves obtained with gyroaveraging with those, for which finite gyroradius effects have not been included. In the latter case, the decay is even weaker than for the beam ion case, indicating that only the increased orbit decorrelation time is at work, but not the reduced $E \times B$ drift. Although orbit averaging is clearly not valid, a peak can be observed at $E=40 \mathrm{keV}$ for the case with the background drift, reflecting the existence of a resonance between the particle curvature drift and the drift of the fluctuations. We attribute this behavior to the fact that only for that case, $\tau_{\text {drop }} \gg \tau^{\text {orbit }}$, whereas a small influence of the drift barrier may remain for the other energies.

As in the previous section, we now want to devise a quantitative formula for $D(E)$. To this aim, we again approximate the diffusivity in the large energy limit by the expression $D \sim\left(V^{\text {eff }}\right)^{2} \tau^{\text {orbit }} / 3$ where the factor $1 / 3$ follows from the observation that in practice, the decorrelation occurs already before the nominal decorrelation time is reached. With Eq. (11), we then find

$$
D=\frac{\left(V^{\mathrm{eff}}\right)^{2} \lambda_{c}^{\mathrm{eff}} R_{0}^{0.5} r_{0}^{0.5} B_{0} e}{3 \sqrt{2} \eta \sqrt{1-\eta^{2}} E} \approx \frac{\left\langle\left(\phi^{\mathrm{eff}}\right)^{2}\right\rangle R_{0}^{0.5} r_{0}^{0.5} e}{3 \sqrt{2} \lambda_{c}^{\mathrm{eff}} \eta \sqrt{1-\eta^{2}} B_{0} E} .
$$

In the case without gyroaveraging, we simply set $V^{\mathrm{eff}}=V$ and $\lambda_{c}^{\mathrm{eff}}=\lambda_{c}$. Inserting the nominal physical parameters yields $D=181 \mathrm{~m}^{2} / \mathrm{s}(E / \mathrm{keV})^{-1}$. Fig. 9 shows that this approach fits the respective simulation results quite well in the high energy limit. To obtain a realistic description of the behavior for low- $\eta$ particles, we have to calculate the effective (i.e., gyroaveraged) values. In the large gyroradius limit, they are already known from Eq. (13). Expressing the gyroradius as $\rho_{g}=\sqrt{1-\eta^{2}} \sqrt{2 E m} /(e B)$ and inserting the effective values into Eq. (23), we get

$$
D=\frac{1.73 V^{2} \lambda_{c}^{2} e^{2} B^{2} R_{0}^{0.5} r_{0}^{0.5}}{24 \pi^{0.5} \eta\left(1-\eta^{2}\right) m^{0.5} E^{1.5}}=\frac{1.73\left\langle\phi^{2}\right\rangle e^{2} R_{0}^{0.5} r_{0}^{0.5}}{24 \pi^{0.5} \eta\left(1-\eta^{2}\right) m^{0.5} E^{1.5}}
$$

Employing again the nominal physical parameters, we find $D=607 /(E / \mathrm{keV})^{1.5}$, which, as can be inferred from Fig. 9, matches the simulation results quite well in the large energy limit. At this point, we have to recall that the validity condition for this approach is that both the gyroradius and the orbit radius exceed $\lambda_{c}$, such that the effective $E \times B$ drift approach applies, and that the orbit decorrelation is dominant. In Eq. (24), a $1 / E$ decay is produced by the orbit decorrelation, and an additional $E^{-0.5}$ decay comes from the gyration. We note in passing that for our beam ion case $(\eta=0.99)$, finite gyroradius effects become relevant only for energies exceeding $1 \mathrm{MeV}$, and that a $E^{-1.5}$ decay may also be expected in this "ultra fast" regime.

Replacing the parameters characterizing the background potential by dimensionless values, as we have also done in the previous section, one obtains

$$
D \approx \frac{1.73\left\langle\phi_{\text {dim.less }}^{2}\right\rangle R_{0}^{0.5} r_{0}^{0.5} m^{0.5}\left(k_{B} T_{e}\right)^{3}}{24 \pi^{0.5} \eta\left(1-\eta^{2}\right) e^{2} B_{0}^{2} L_{\perp}^{2} E^{1.5}} \sim \frac{m^{0.5}\left(k_{B} T_{e}\right)^{3}}{e^{2} B_{0}^{2} R_{0} E^{1.5}}=\left(\frac{E}{k_{B} T_{e}}\right)^{-1.5} D_{\text {gyroBohm }}
$$


where we have assumed $r_{0} \sim R_{0} \sim L_{\perp}$. We thus find a slightly faster decay than in the large- $\eta$ case.

\section{SUMMARY AND CONCLUSIONS}

In the present paper, we have studied - in the passive tracer limit - the interaction of energetic ions with electrostatic microturbulence for an idealized ITER-like tokamak. In this context, several details of both the particle orbit parameters and the properties of the turbulent fluctuations turn out to be important. Nevertheless, it is possible to understand and quantitatively predict the behavior of fast particles both in the high-energy regime, $E / T_{e} \gg 1$, as well as in the moderate-energy regime, $1 \lesssim E / T_{e} \lesssim 10$.

A first crucial insight is that while, in principle, regimes exist for which it is possible to average the $E \times B$ drift motion over one drift orbit time, such a procedure is usually not valid (except maybe for low magnetic shear). In the former case, a particle follows the orbit averaged structures, and the existence of a drift barrier (caused by the poloidal drift of the particles) leads to a strong reduction of the diffusivity with increasing particle energy. In the latter case, however, a particle decorrelates from its original position in the turbulent field already along its orbit. The respective time scale is typically smaller than the autocorrelation time of the fluctuations or the drop time associated with the drift motion. This explains why the observed reduction of the diffusivity is weaker than expected based on orbit averaging arguments. For beam ions, a $\left(E / T_{e}\right)^{-1}$ fall-off has been found analytically as well as numerically, whereas for ions with a small parallel velocity component, a $\left(E / T_{e}\right)^{-1.5}$ decrease was found, due to additional finite gyroradius effects.

Besides the high energy limit, we also studied the behavior of particles with $1 \lesssim E / T_{e} \lesssim 10$. It turned out that the transport of such moderately suprathermal particles may remain on a level comparable to that of thermal particles. This can be attributed to the existence of resonances between the particle drifts and the diamagnetic drift of the background turbulence. In the case of resonance, orbit averaging may become valid, whereas at the same time, the drift barrier does not exist any more - two effects which work together synergistically. The parallel decorrelation was found to be of minor relevance for all particle energies, since decorrelation due to the perpendicular motion is typically faster by up to one order of magnitude.

By means of these findings and insights, one is able to explain in detail the simulation results reported in Ref. [9]. Moreover, they can be applied to try to understand and interpret recent experimental observations [11] concerning the efficiency of neutral beam injection. A thorough discussion of the latter problem will be presented elsewhere.

\section{Acknowledgements}

We would like to thank T. Dannert and S. Günter for useful discussions, as well as E. Strumberger and E. Schwarz for help with the GOURDON code. 
[1] G. Manfredi and R. O. Dendy, Phys. Rev. Lett. 76, 4360 (1996).

[2] G. Manfredi and R. O. Dendy, Phys. Plasmas 4, 628 (1997).

[3] S. V. Annibaldi, G. Manfredi, and R. O. Dendy, Phys. Plasmas 9, 791 (2002).

[4] M. Vlad and F. Spineanu, Plasma Phys. Control. Fusion 47, 281 (2005).

[5] M. Vlad, F. Spineanu, S. I. Itoh, M. Yagi, and K. Itoh, Plasma Phys. Control. Fusion 47, 1015 (2005).

[6] T. Hauff and F. Jenko, Phys. Plasmas 13, 102309 (2006).

[7] T. Hauff and F. Jenko, Phys. Plasmas 14, 092301 (2007).

[8] C. Estrada-Mila, J. Candy, and R. E. Waltz Phys. Plasmas 13, 112303 (2006).

[9] T. Dannert, S. Günter, T. Hauff, F. Jenko, and P. Lauber, Turbulent transport of energetic ions, to appear in Phys. Plasmas.

[10] C. Angioni and A. G. Peeters, Phys. Plasmas 15, 052307 (2008).

[11] S. Günter, G. Conway, S. daGraça, H. -U. Fahrbach, C. Forest, M. Garcia Muñoz, T. Hauff, J. Hobirk, V. Igochine, F. Jenko, K. Lackner, P. Lauber, P. McCarthy, M. Maraschek, P. Martin, E. Poli, K. Sassenberg, E. Strumberger, G. Tardini, E. Wolfrum, H. Zohm, and ASDEX Upgrade Team, Nucl. Fusion 47, 920 (2007).

[12] W. W. Heidbrink, C. W. Barnes, G. W. Hammett, Y. Kusama, S. D. Scott, M. C. Zarnstorff, L. C. Johnson, D. McCune, S. S. Medley, H. K. Park, A. L. Roquemore, J. D. Strachan, and G. Taylor, Phys. Fluids B 3, 3167 (1991).

[13] www.iter.org

[14] E. A. Frieman and L. Chen, Phys. Fluids 25, 502 (1982).

[15] M. Vlad, F. Spineanu, J. H. Misguich, and R. Balescu, Phy. Rev. E 58, 7359 (1998).

[16] J. Wesson, Tokamaks, (Clarendon Press, Oxford, 1987), Chap.3.

[17] H. E. Mynick and J. A. Krommes Phys. Rev. Lett. 43, 1506 (1979).

[18] J. R. Myra, P. J. Catto, H. E. Mynick, and R. E. Duvall, Phys. Fluids B 5, 1160 (1993).

[19] G. Rewoldt, Phys. Fluids 31, 3727 (1988).

[20] G. Rewoldt, Nucl. Fusion 31, 2333 (1991).

[21] T. Fülöp, private communication (2008).

[22] R. Basu, T. Jessen, V. Naulin, and J. J. Rasmussen, Phys. Plasmas 10, 2696 (2003).

[23] M. D. Kruskal and R. M. Kulsrud, Phys. Fluids 1, 265 (1958).

[24] J. M. Greene and J. L. Johnson, Phys. Fluids 5, 510 (1962).

[25] X. Lapillonne and S. Brunner, private communication.

[26] M. A. Beer, S. C. Cowley, and G. W. Hammett, Phys. Plasmas 2, 2687 (1995).

[27] F. Jenko, W. Dorland, M. Kotschenreuther, and B. N. Rogers, Phys. Plasmas 7, 1904 (2000).

[28] T. Dannert and F. Jenko, Phys. Plasmas 12, 072309 (2005).

[29] T. M. Antonsen, Jr. , J. F. Drake, P. N. Guzdar, A. B. Hassan, Y. T. Lau, C. S. Liu, and S. V. Novakowskii, Phys. Plamas 3, 2221 (1996).

[30] R. G. Littlejohn, J. Plasma Phys. 29, 111 (1983).

[31] C. Gourdon, Programme optimisé de calculs numériques dans les configurations magnétiques toroidales, CEN, Fontenay aux Roses (1970).

[32] G. I. Taylor, Proc. Lond. Math. Soc. 20, 196 (1920).

[33] S. J. Zweben and S. S. Medley, Phys. Fluids B 1, 2058 (1989).

[34] A. J. Wooton, B. A. Carreras, H. Matsumoto, K. McGuire, W. A. Peebles, C. P. Ritz, P. W. Terry, and S. J. Zweben, Phys. Fluids B 2, 2879 (1990).

[35] H. Thomsen, M. Endler, J. Bleuel, A. V. Chankin, S. K. Erents, and G. F. Matthews, Phys. Plasmas 9, 1233 (2002).

[36] N. Mahdizadeh, F. Greiner, T. Happel, A. Kendl, M. Ramisch, B. D. Scott, and U. Stroth, Plasma Phys. Control. Fusion 49, 1005 (2007).

[37] A. N. Kaufman, Phys. Fluids 15, 1063 (1972).

[38] H. E. Mynick and A. H. Boozer, Phys. Plasmas 12, 062513 (1995). 


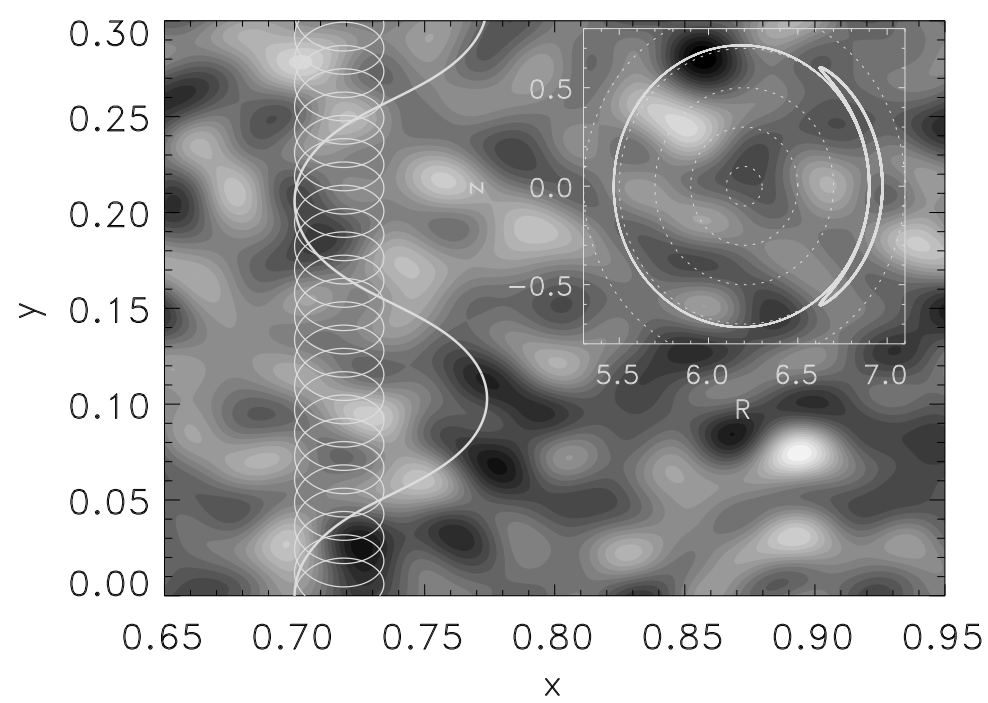

FIG. 1: Particle orbits (in a fluctuating electrostatic potential) in field-aligned coordinates (left-hand side) and in cylindrical coordinates (embedded). The bold line denotes a trapped particle with $E=100 \mathrm{keV}$ and $\eta=0.2$, while the thin line denotes a passing particle with $E=100 \mathrm{keV}$ and $\eta=0.99$. Some magnetic flux surfaces are shown for comparison.

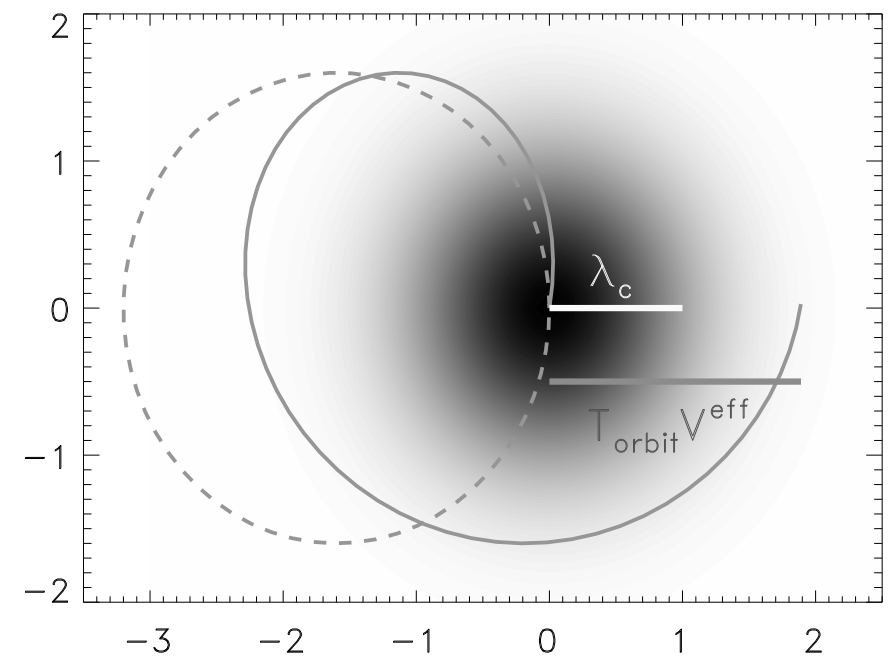

FIG. 2: The dashed line denotes a circle over which the potential is (orbit-)averaged for a particle starting at the origin, while the solid line denotes a real particle trajectory with a large $E \times B$ drift velocity $\bar{V}^{\text {eff }}$. After one period, the particle is displaced by $T_{\text {orbit }} \bar{V}^{\text {eff }}$ from the origin as well as from the corresponding point on the dashed curve. Therefore, if the particle does not return into the correlated zone [in the background, the autocorrelation function $\langle\phi(0) \phi(\mathbf{x})\rangle$ of an isotropic stochastic potential with correlation length $\lambda_{c}$ is plotted], orbit averaging is not valid. 


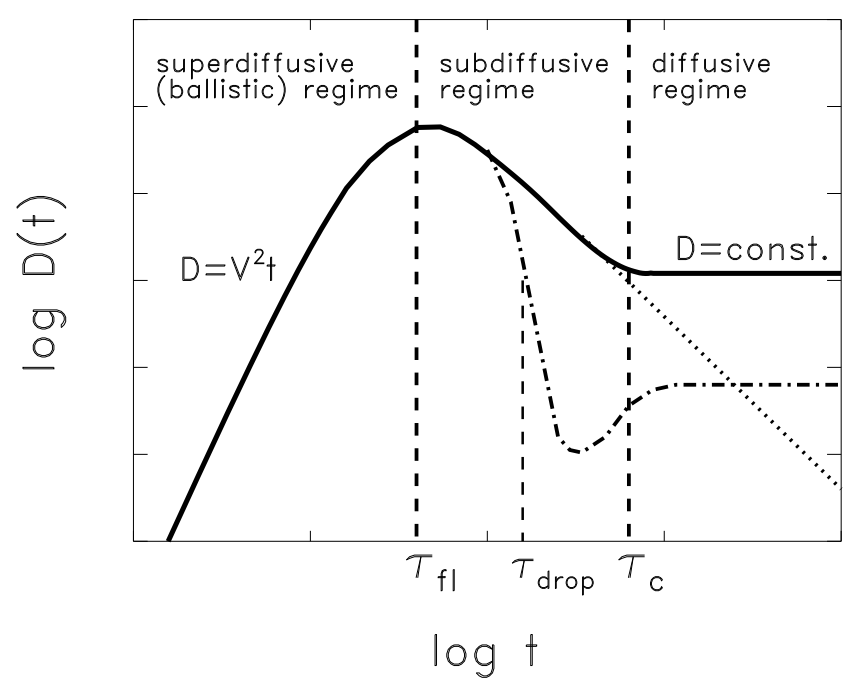

FIG. 3: Schematic time evolution of the running diffusion coefficient in two dimensions. Solid line: time dependent electrostatic potential without background drift. Dotted line: static potential without background drift. Dashed dotted line: time dependent potential with background drift and $\tau_{\text {drop }}<\tau_{c}$. Finite gyroradius effects would shift the solid curve downwards $\left(V^{\mathrm{eff}}<V\right)$. Moreover, the maximum would be shifted to the right $\left(\tau_{\mathrm{ff}}^{\text {eff }}>\tau_{\mathrm{ff}}\right)$.

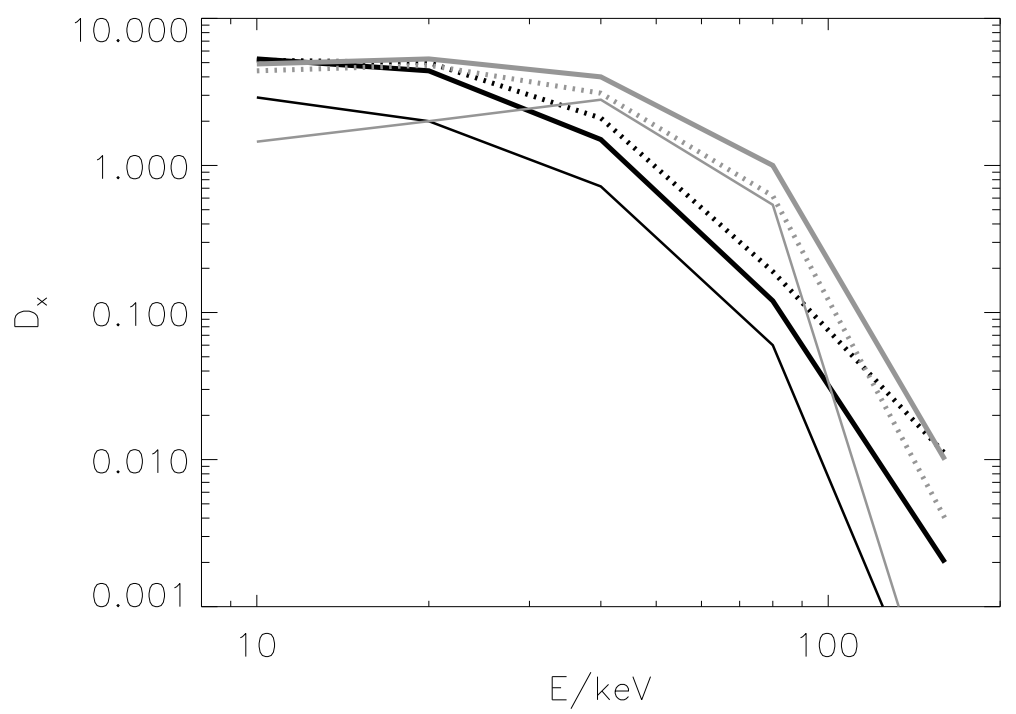

FIG. 4: Radial diffusion coefficient $D_{x}$ for different particle energies $E$ and $\eta=0.99$ in a shearless magnetic field. Black lines: no background drift $\left(v_{\mathrm{dr}}=0\right)$. Grey lines: background drift $\left(v_{\mathrm{dr}}=300 \mathrm{~m} / \mathrm{s}\right)$. Bold solid lines: particles in a tokamak (Eq. (7)). Bold dotted lines: simple 2D model (Eq. (18)). Thin solid lines: orbit averaging in the simple 2D model. 


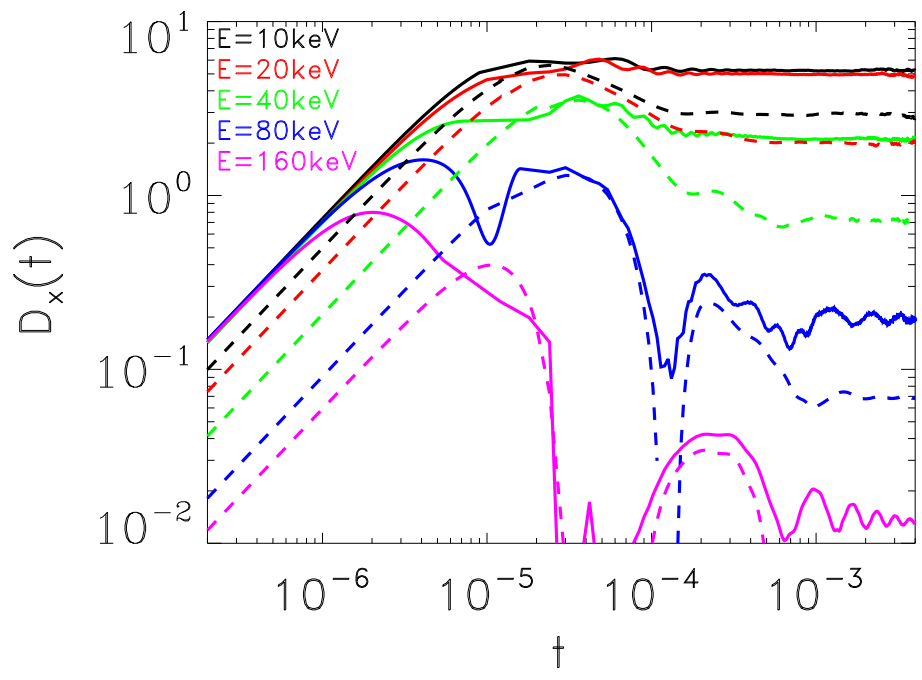

FIG. 5: (Color online) Running radial diffusion coefficient $D_{x}(t)$ for different beam energies and $v_{\mathrm{dr}}=0$. Solid lines: forced orbit motion according to Eq. (18). Dashed lines: orbit averaging. The saturation values are the same as in Fig. 4.

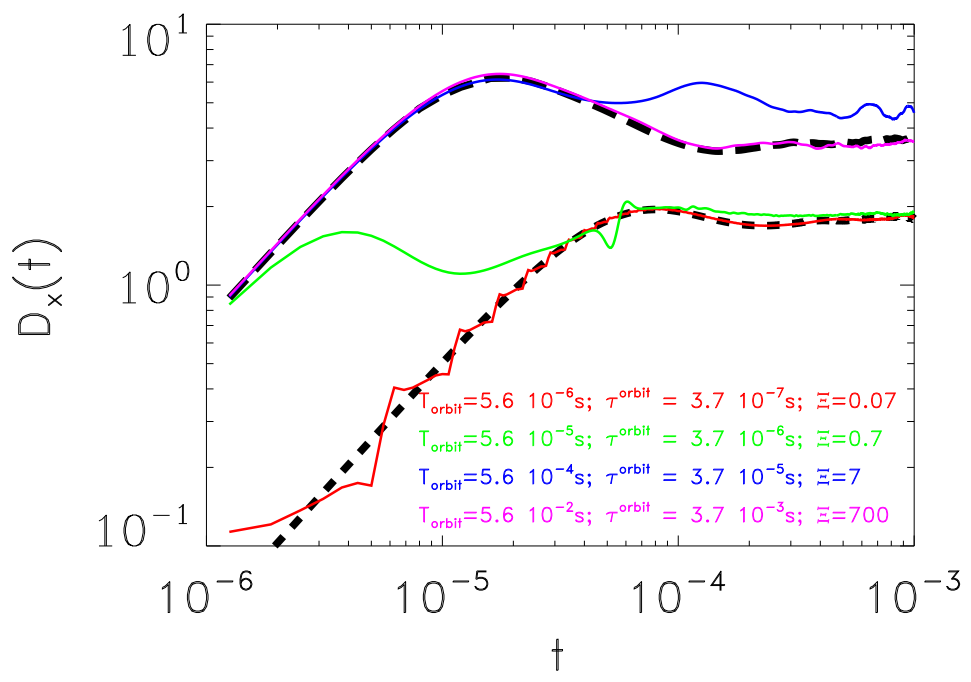

FIG. 6: (Color online) Running radial diffusion coefficient $D_{x}(t)$ for different orbit cycle durations. Here, the orbit radius is kept fixed at $\Delta x / 2 \equiv 0.04 \mathrm{~m}$, and $v_{y} \equiv v_{\mathrm{dr}} \equiv 0$. Lower dashed line: orbit averaging. Upper dashed line: no orbit motion. Solid lines: full $2 \mathrm{D}$ orbit motion. A significant difference in the time evolution for the regimes $\Xi_{\text {o.a. }}<1$ and $\Xi_{\text {o.a. }}>1$ can be observed. 


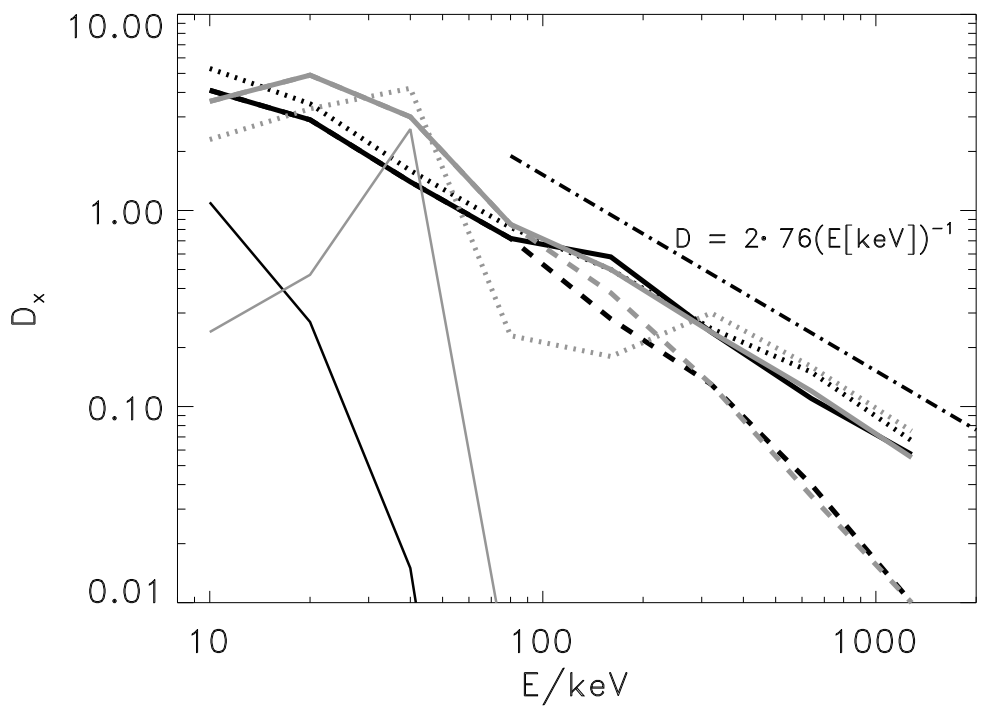

FIG. 7: Radial diffusion coefficient $D_{x}$ for different particle energies $E$ and $\eta=0.99$ in a sheared magnetic field. Black lines: no background drift $\left(v_{\mathrm{dr}}=0\right)$. Grey lines: background drift $\left(v_{\mathrm{dr}}=1 \mathrm{~km} / \mathrm{s}\right)$. Bold solid lines: particles in an annulus. Bold dashed lines: particles an annulus consisting of 10 flux tubes with periodic boundary conditions. Bold dotted lines: simple 2D model (Eq. (18)). Thin solid lines: orbit averaging in the simple 2D model. Dashed-dotted line: analytic approach. The dashed-dotted curve has been multiplied by a factor of two to become distinguishable from the simulation results.

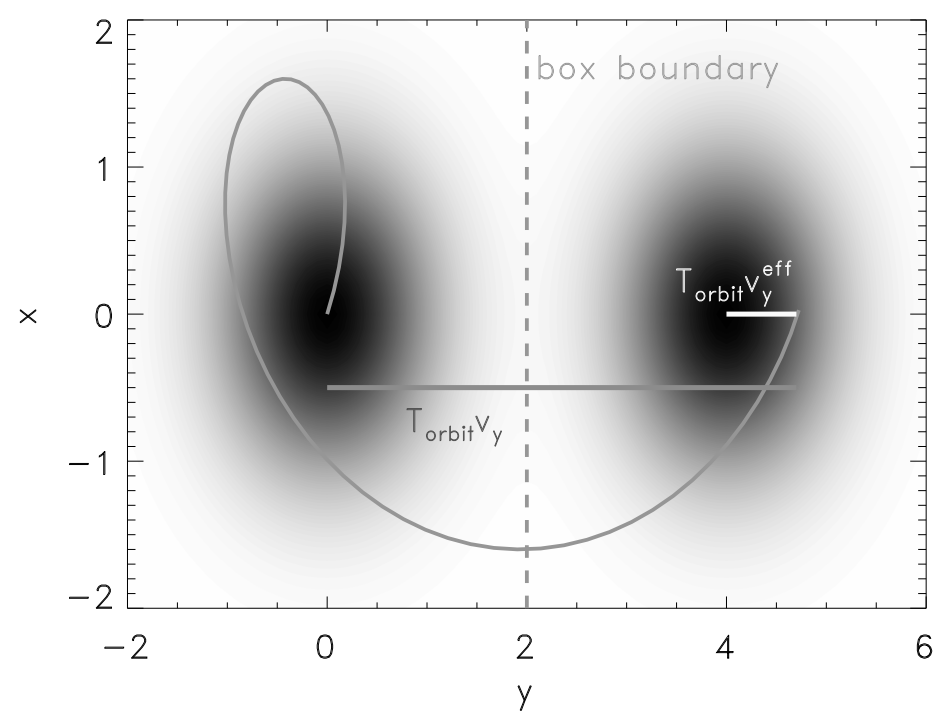

FIG. 8: This figure is similar to Fig. 2. It illustrates the mechanism of unphysical re-correlations if a flux surface is covered by several flux tubes. Here, the real drift velocity $v_{y}$ is replaced by the effective velocity $v_{y}^{\text {eff }}=\left(T_{\text {orbit }} v_{y} \bmod L_{y}\right) / T_{\text {orbit }}$. 


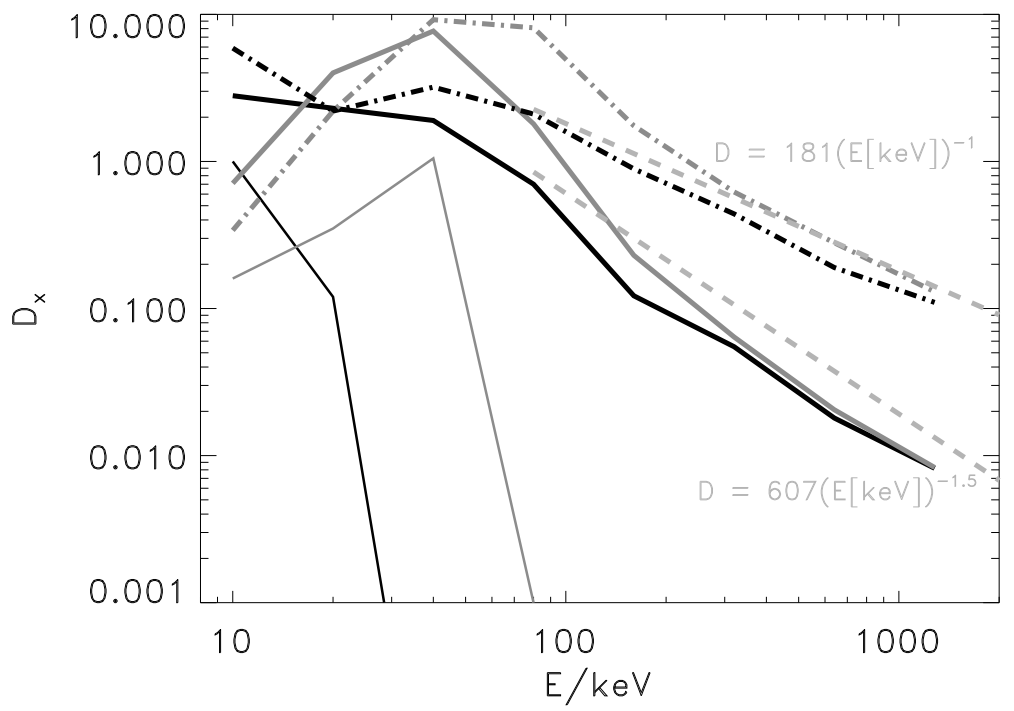

FIG. 9: Radial diffusion coefficient $D_{x}$ for different particle energies $E$ and $\eta=0.2$ in a sheared magnetic field. Dark lines: no background drift $\left(v_{\mathrm{dr}}=0\right)$. Grey lines: background drift $\left(v_{\mathrm{dr}}=1 \mathrm{~km} / \mathrm{s}\right)$. Bold solid lines: particles in a tokamak (Eq. (7)). Finite gyroradius effects are included via gyroaveraging. Bold dashed dotted lines: the same, but without finite gyroradius effects. Thin solid lines: orbit averaging in the simple 2D model. Grey dashed lines: analytic approach with and without gyroaveraging.

\begin{tabular}{|c|c|c|c|c|c|c|c|c|c|}
\hline$E$ & $T_{\text {orbit }}$ & $\Delta x / 2$ & $\Delta y / 2$ & $v_{y}$ & $\Xi_{\text {o.a. }}^{v_{\mathrm{dr}}=0}$ & $\Xi_{\text {o.a. }}^{v_{\mathrm{dr}}}=300 \mathrm{~m} / \mathrm{s}$ & $\tau_{\mathrm{drop}}^{v_{\mathrm{dr}}=0}$ & $\tau_{\text {drop }}^{v_{\mathrm{dr}}=300 \mathrm{~m} / \mathrm{s}}$ & $\tau^{\text {orbit }}$ \\
\hline $10 \mathrm{keV}$ & $5.6 \cdot 10^{-5}$ & $0.0055 \mathrm{~m}$ & $0.0050 \mathrm{~m}$ & $68 \mathrm{~m} / \mathrm{s}$ & 2.0 & 2.0 & $4.8 \cdot 10^{-4}$ & s $1.4 \cdot 10^{-4}$ & $2.8 \cdot 10^{-5} \mathrm{~s}$ \\
\hline $20 \mathrm{keV}$ & $4.0 \cdot 10^{-5}$ & $0.0076 \mathrm{n}$ & $0.0075 \mathrm{~m}$ & $136 \mathrm{~m} / \mathrm{s}$ & 1.2 & 1.2 & $2.4 \cdot 10^{-4}$ & s $2.0 \cdot 10^{-4}$ & $1.4 \cdot 10^{-5} \mathrm{~s}$ \\
\hline $40 \mathrm{keV}$ & $2.8 \cdot 10^{-5}$ & $0.0110 \mathrm{~m}$ & $0.0105 \mathrm{~m}$ & $273 \mathrm{~m} / \mathrm{s}$ & 0.7 & 0.7 & $1.2 \cdot 10^{-4}$ & $1.2 \cdot 10^{-3}$ & $6.6 \cdot 10^{-6}$ \\
\hline $80 \mathrm{keV}$ & $2.0 \cdot 10^{-5}$ & $0.0155 \mathrm{~m}$ & $0.0148 \mathrm{~m}$ & $545 \mathrm{~m} / \mathrm{s}$ & 0.6 & 0.4 & $6.0 \cdot 10^{-5}$ & $1.3 \cdot 10^{-4}$ & $3.4 \cdot 10^{-6}$ \\
\hline $160 \mathrm{keV}$ & $1.4 \cdot 10^{-5}$ & $0.0215 \mathrm{~m}$ & $0.0205 \mathrm{~m}$ & $1092 \mathrm{~m} / \mathrm{s}$ & 0.9 & 0.6 & $3.0 \cdot 10^{-5}$ & s $4.1 \cdot 10^{-5}$ & $1.7 \cdot 10^{-6}$ \\
\hline
\end{tabular}

TABLE I: Characteristic orbit parameters for beam ions $(\eta=0.99)$ in an ITER-like tokamak with $R_{0}=6.2 \mathrm{~m}, B_{0}=5.3 \mathrm{~T}$, and $q(r) \equiv 1.4$. The plasma/turbulence parameters are $T=10 \mathrm{keV}, \tau_{c}=1.8 \cdot 10^{-4} \mathrm{~s}, \lambda_{c}=0.0164 \mathrm{~m}$, and $V=900 \mathrm{~m} / \mathrm{s}$. For the calculation of $\Xi_{\text {o.a. }}, \bar{V}^{\text {eff }}$ has been determined according to Eq. (13) with $\rho_{g}$ replaced by $\Delta x / 2$. Finite curvature drift velocities $v_{y}$ are found although the magnetic shear vanishes.

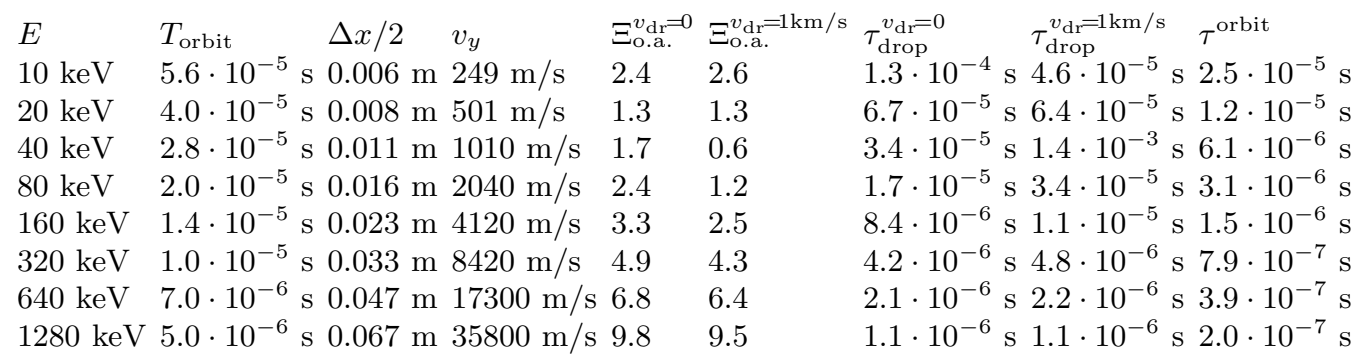

TABLE II: Characteristic orbit parameters for beam ions $(\eta=0.99)$ in an ITER-like tokamak with $R_{0}=6.2 \mathrm{~m}, B_{0}=5.3 \mathrm{~T}$, and $q(r)=0.5 r^{2}+1.25$. The plasma/turbulence parameters are as usual. For the calculation of $\Xi_{\text {o.a. }}, \bar{V}^{\text {eff }}$ has been determined according to Eq. (13) with $\rho_{g}$ replaced by $\Delta x / 2$. All parameters are derived from simulations; they are in good agreement with the analytical expressions from Eq. (9). 


\begin{tabular}{|c|c|c|c|c|c|c|c|}
\hline$E$ & $T_{\text {orbit }}$ & $\Delta x / 2$ & $\rho_{g}$ & $v_{y}$ & $\Xi_{\text {o.a. }}^{v_{\mathrm{dr}}=0}$ & $\Xi_{\text {O.a. }}^{v}=1 \mathrm{~km} / \mathrm{s}$ & $\tau_{\mathrm{drop}}^{v_{\mathrm{dr}}=1 \mathrm{~km} / \mathrm{s}} \quad \tau^{\text {orbit }}$ \\
\hline $0 \mathrm{keV}$ & $2.6 \cdot 10^{-4}$ & $0.011 \mathrm{~m}$ & $0.0038 \mathrm{~m}$ & $237 \mathrm{~m} / \mathrm{s}$ & 5.3 & 12.1 & $1.4 \cdot 10^{-4} \mathrm{~s} 4.3 \cdot 10^{-5} \mathrm{~s} 6.8 \cdot 10^{-5} \mathrm{~s}$ \\
\hline $0 \mathrm{keV}$ & $1.8 \cdot 10^{-4}$ & $0.016 \mathrm{~m}$ & $0.0054 \mathrm{~m}$ & $472 \mathrm{~m} / \mathrm{s}$ & 5.2 & 5.8 & $7.5 \cdot 10^{-5}$ s 6.2 \\
\hline $0 \mathrm{keV}$ & $1.3 \cdot 10^{-4}$ & $0.023 \mathrm{~m}$ & $0.0076 \mathrm{~m}$ & $938 \mathrm{~m} / \mathrm{s}$ & 7.4 & 1.3 & $3.5 \cdot 10^{-5}$ s $5.5 \cdot 10^{-4}$ s $1.7 \cdot 10^{-5}$ \\
\hline $\mathrm{keV}$ & $9.0 \cdot 10^{-5}$ & $0.033 \mathrm{~m}$ & $0.011 \mathrm{~m}$ & $1860 \mathrm{~m} / \mathrm{s}$ & 10.3 & 4.8 & $1.7 \cdot 10^{-5} \mathrm{~s} 3.7 \cdot 10^{-5} \mathrm{~s} 8.4 \cdot 10^{-6}$ \\
\hline $50 \mathrm{keV}$ & $6.4 \cdot 10^{-5}$ & $0.047 \mathrm{~m}$ & $0.015 \mathrm{~m}$ & $3680 \mathrm{~m} / \mathrm{s}$ & 4.7 & 0.8 & $8.7 \cdot 10^{-6}$ s $1.2 \cdot 10^{-5}$ s $4.2 \cdot 10^{-6}$ \\
\hline $\mathrm{V}$ & $4.5 \cdot 10^{-5}$ & $.067 \mathrm{~m}$ & $0.021 \mathrm{~m}$ & 7270 & 20.6 & 17.9 & $4.4 \cdot 10^{-6}$ s $5.0 \cdot 10^{-6}$ \\
\hline & $3.2 \cdot 10^{-}$ & $096 \mathrm{~m}$ & $0.031 \mathrm{~m}$ & 14300 & 9.4 & 27.4 & $3 \cdot 10^{-6} \mathrm{~s} 1.1 \cdot 10^{-6}$ \\
\hline 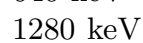 & $2.3 \cdot 10^{-5}$ & $.139 \mathrm{~m}$ & $0.042 \mathrm{~m}$ & 28000 & 1.2 & 40.8 & $.1 \cdot 10^{-6}$ s $5.4 \cdot 10^{-2}$ \\
\hline
\end{tabular}

TABLE III: Characteristic orbit parameters for ions with $\eta=0.2$ in an ITER-like tokamak with $R_{0}=6.2 \mathrm{~m}, B_{0}=5.3 \mathrm{~T}$, and $q(r)=0.5 r^{2}+1.25$. The plasma/turbulence parameters are as usual. All parameters are derived from simulations; they are in good agreement with the analytical expressions from Eq. (11). The finite gyroradius of the particles has been taken into account via gyroaveraging. 